\title{
On Truncated Zeghdoudi Distribution: Posterior Analysis under Different Loss Functions for Type II Censored Data
}

\author{
Talhi Hamida*1, Aiachi Hiba ${ }^{2}$
}

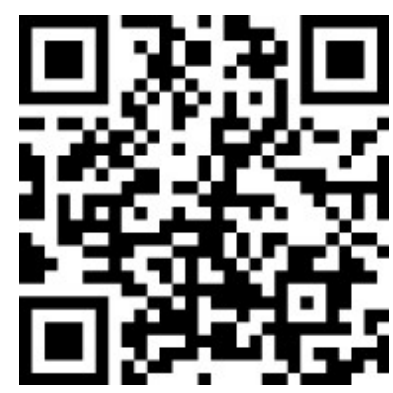

${ }^{*}$ Corresponding author

1. Probability Statistics laboratory; Badji Mokhtar University, Algeria, talhihamida@yahoo.fr.

2. Probability Statistics laboratory; Badji Mokhtar University, Algeria, aiachihiba@yahoo.com.

\begin{abstract}
We perform a Bayesian analysis of the upper truncated Zeghdoudi distribution based on type II censored data. Using various loss functions including the generalized quadratic, entropy and Linex functions, we obtain Bayes estimators and the corresponding posterior risks. As tractable analytical forms of these estimators are out of reach, we propose Markov chain Monte-Carlo (MCMC) based simulation approach to study their performance. Moreover, given initial values for the parameters of the model, we obtain maximum likelihood estimators. Furthermore, we compare their performance with those of the Bayesian estimators using Pitman's closeness criterion and integrated mean square error. Finally, we illustrate our approach through an example with real data.
\end{abstract}

keywords: Truncated Zeghdoudi distribution, Bayes estimators, generalized quadratic loss function, Linex loss function, posterior risk, Metropolis-Hastings algorithm, Pitman closeness criterion.

\section{Introduction}

Truncation is the process of excluding and omitting all the values that lay outside predetermined bounds in a statistical experiment. The remaining data points inside these bounds are called truncated data.

A random variable $X$ is upper (lower) truncated, at a given threshold level $c$, if only the outcomes of $X$ for which $X \leq c(X>c)$ are considered, i.e. we omit all the values of $X$ for which $X>c(X \leq c)$. For example, age is lower truncated, since it starts at Zero $(c=0)$.

Truncated distributions are used in a wide range of applications. Recent work includes Bantan et al. (2019) who introduced the Truncated Inverted Kumaraswamy generated family of distributions and discussed its applications. Aldahlan et al. (2020) have introduced the truncated Cauchy power family of distributions with Inference and its Applications. Aouf and Chadli (2017) considered the Bayesian inference under type II censored data of the generalized Lindley distribution. Boudjerda et al. (2016) also considered the Bayesian analysis under type II censored data of right truncated Weibull distribution. Singh et al. (2014) have introduced the truncated version of the Lindley distribution and discussed its properties. Finally, Balakrishnan and Mitra (2012) applied the EM algorithm to study the performance of the parameters' estimators of the left truncated Weibull distribution under right censored data.

The Lindley distribution with one parameter $\theta$ is a mixture of exponential $(\theta)$ and gamma $(2, \theta)$ distributions. 
It is widely used in modelling lifetime data including the series of papers by Zeghdoudi and Nedjar (2016a, 2016b, 2016c) where it is shown that it fits well a large class of real data sets. Messaadia and Zeghdoudi (2018) suggested a one parameter exponential family of distribution which is based on mixtures of gamma $(2, \theta)$ and gamma $(3, \theta)$ distribution, known as the Zeghdoudi distribution and showed that it fits well lifetime data sets where the Lindley distribution gave poor fit.

In this paper, we propose a Bayesian analysis of the new upper truncated Zeghdoudi distribution. We first introduce the upper-truncated Zeghdoudi distribution (UTZ) which depends on two parameters. Then we derive the maximum likelihood (ML) estimators of these parameters for type II censored data. Furthermore, we derive the Bayesian estimators of these parameters under the generalized quadratic (GQ), the entropy and the Linex loss functions. We perform a simulation experiment to study the behaviour of the proposed estimators and compare them with the ML estimator using Pitman's closeness criterion. Finally, we compute the integrated mean square error (IMSE) for the three Bayesian estimators.

The rest of the paper is organized as follows. In section 2, we derive the truncated version of the Zeghdoudi distribution. The estimation of the parameters is displayed in section 3. Monte-Carlo simulation results are presented in section 4 . In section 5 , to illustrate the obtained results, we present an example based on real data. Finally, we conclude the paper in section 6 .

\section{The upper truncated Zeghdoudi distribution}

The probability density function of the Zeghdoudi distribution (see Messaadia and Zeghdoudi (2018)) is

$$
f_{Z D}(x, \theta)=\frac{\theta^{3} x(1+x) e^{-\theta x}}{2+\theta}, \quad x, \theta>0,
$$

and its cumulative function is

$$
F_{Z D}(x)=\frac{1-\left(x^{2} \theta^{2}+\theta(\theta+2) x+\theta+2\right)}{(\theta+2) e^{-\theta x}}, \quad x, \theta>0 .
$$

The probability density function of the upper truncated Zeghdoudi distribution with the parameter $\beta>0$, is given by

$$
f_{U T Z}(x, \theta)=f_{Z D}(x, \theta \mid X \leq \beta)=\frac{f_{Z D}(x, \theta)}{F_{Z D}(\beta)},
$$

which, in view of (1) and (2), is explicitly given by the formula

$$
\left.f_{U T Z}(x, \theta)=\left(\frac{\theta^{3} x(1+x)}{(\theta+2) e^{\beta \theta}-\left(\beta^{2} \theta^{2}+\theta(\theta+2) \beta+\theta+2\right.}\right)\right) e^{(-\theta x+\beta x)}, \quad \theta \leq x \leq \beta .
$$

Thus, the cumulative function is

$$
F_{U T Z}(x)=\left(\frac{(\theta+2) e^{\theta x}-x^{2} \theta^{2}-\theta(\theta+2) x-\theta-2}{(\theta+2) e^{\beta \theta}-\beta^{2} \theta^{2}-\theta(\theta+2) \beta-\theta-2}\right) e^{(-x \theta+\beta \theta)} .
$$

\section{Estimation of the unknown parameters}

\subsection{Maximum likelihood estimation}

Let the sample $\left(x_{1}, x_{2}, \ldots, x_{n}\right)$ be generated from upper the truncated Zeghdoudi model, assuming that the data is type II censored, i.e., we only observe $\left(x_{1}, x_{2}, \ldots, x_{m}\right)$, for a given $m \in\{1,2, \ldots, n\}$. The likelihood function is

$$
L(\theta, \beta \mid X)=\frac{n !}{(n-m) !} \prod_{i=1}^{m} f_{U T Z}\left(x_{i}, \theta, \beta\right)\left[\left(1-F_{U T Z}\left(x_{m}\right)\right)\right]^{n-m}
$$


By setting

$$
\begin{aligned}
& A=n ! /(n-m) ! \\
& B=\left[(\theta+2) \exp (\beta \theta)-\left(\beta^{2} \theta^{2}+\theta(\theta+2) \beta+\theta+2\right)\right]^{-n}, \\
& C=\left[\beta^{2} \theta^{2}+\theta(\theta+2) \beta+\theta+2+\left(x_{m} \theta^{2}+(\theta+2) x_{m}+\theta+2\right) e^{\left(-x_{m} \theta+\beta \theta\right)}\right],
\end{aligned}
$$

the likelihood function reads

$$
L(\theta, \beta \mid X)=A B^{-n} C^{n-m} \theta^{3 m} \exp (m \beta \theta) \prod_{i=1}^{m} x_{i}\left(1+x_{i}\right) \exp \left(\theta x_{i}\right) .
$$

The corresponding logarithm is

$$
\begin{aligned}
l(\theta, \beta \mid X) & =\ln (A)-n \ln (B)+(n-m) \ln (C)+3 m \ln (\theta)+m \beta \theta \\
& +\sum_{i=1}^{m} \ln \left(x_{i}\right)+\sum_{i=1}^{m} \ln \left(1+x_{i}\right)+\theta \sum_{i=1}^{m} x_{i} .
\end{aligned}
$$

The solution of the following non-linear system yields the maximum likelihood estimators $\theta_{M L E}$ and $\beta_{M L E}$ of the parameters $\theta, \beta$, respectively.

$$
\left\{\begin{array}{l}
\frac{\partial}{\partial \theta} l(\theta, \beta \mid X)=-n \frac{B_{1}}{B}+(n-m) \frac{C_{1}}{C}+\frac{3 m}{\theta}+m \beta+\sum_{i=1}^{m} x_{i}, \\
\frac{\partial}{\partial \beta} l(\theta, \beta \mid X)=-n \frac{B_{2}}{B}+(n-m) \frac{C_{2}}{C}+m \theta
\end{array}\right.
$$

where

$$
\begin{aligned}
B_{1}= & \frac{\partial B}{\partial \theta}=e^{\beta \theta}[\beta(\theta+2)+1]-2 \beta^{2} \theta-2 \beta(\theta+1)-1 \\
C_{1}= & \frac{\partial C}{\partial \theta}=-\left[2 \beta^{2} \theta+2 \beta(\theta+1)+1+\left(2 x_{m}^{2} \theta+2 x_{m}(\theta+1)+1\right) e^{\left(-x_{m} \theta+\beta \theta\right)}\right. \\
& \left.+\left(\beta-x_{m}\right)\left(x_{m}^{2} \theta^{2}+\theta(\theta+2) x_{m}+\theta+2\right) e^{\left(-x_{m} \theta+\beta \theta\right)}\right] \\
B_{2}= & \frac{\partial B}{\partial \beta}=\theta(\theta+2) \exp (\beta \theta)-\left(2 \beta \theta^{2}+\theta(\theta+2)\right) \\
C_{2}= & \frac{\partial C}{\partial \beta}=-\left(2 \beta \theta^{2}+\theta(\theta+2)+\theta\left(x_{m}^{2}+\theta^{2}+\theta(\theta+2) x_{m}+\theta+2\right) e^{\left(-x_{m} \theta+\beta \theta\right)}\right.
\end{aligned}
$$

The solution of the system (7) seems analytically intractable. We will rely on numerical methods to obtain approximate solutions. We will use the $R$ package $B B$ to obtain the approximate value of the maximum likelihood estimators $\theta_{M L E}$ and $\beta_{M L E}$ of the parameters $\theta, \beta$. The $\mathrm{R}$ package $B B$ is successfully used for solving non-linear system of equations; see Varadhan and Gilbert (2010).

\subsection{Bayesian Estimation under different loss functions}

In the Bayesian approach, we consider the unknown parameters to be random variables instead of fixed constants unlike in the classical approach. For that matter the variation in the parameters allows to assume a prior information in terms of prior distributions on the unknown parameters.

\section{(i) Prior and posterior distributions}

We assume here that the parameters $\theta, \beta$ of the UTZ distribution have independent gamma distributions:

$$
\begin{aligned}
& \pi(\theta)=\frac{a^{b}}{\Gamma(b)} \theta^{b-1} \exp (-a \theta), \quad \beta, \theta>0, a, b>0, \\
& \pi(\beta)=\frac{c^{d}}{\Gamma(d)} \beta^{d-1} \exp (-c \beta), \quad \beta, \theta>0, c, d>0 .
\end{aligned}
$$

where the constants $a, b, c, d$ are called hyper-parameters. There is no objective motivation for choosing the gamma family as prior distributions, except for their flexibility, tractability and for being natural conjugate 
priors for the exponential distributions. Other prior distribution may well be used.

The joint prior distribution of $(\theta, \beta)$ is then

$$
\pi(\theta, \beta)=\frac{a^{b} c^{d}}{\Gamma(b) \Gamma(d)} \beta^{d-1} \theta^{b-1} \exp (-a \theta-c \beta) .
$$

The joint posterior distribution of $(\theta, \beta)$ reads

$$
\pi(\theta, \beta / x)=K B^{-n} C^{n-m} \theta^{b-1+3 m} e^{(-a \theta-c \beta+m \beta \theta)} \prod_{i=1}^{m} x_{i}\left(1+x_{i}\right) e^{\theta x_{i}},
$$

where $K$ is a normalizing constant.

(ii) Loss functions

We obtain the Bayesian estimators under three different loss functions. Namely, the generalized quadratic (GQ), the Linex and the entropy loss function.

Let $\delta$ be the estimator of $\lambda$, the generalized quadratic loss function is defined as $L(\lambda, \delta)=\tau(\lambda)(\lambda-\delta)^{2}$. We assume that $\tau(\lambda)=\lambda^{\alpha-1}$ where $\alpha$ is a selected constant. The Bayesian estimator of $\lambda$ has posterior mean

$$
\hat{\delta}_{G Q}=\frac{E_{\pi}(\tau(\lambda) \lambda)}{E_{\pi}(\tau(\lambda))}
$$

and the associated posterior risk

$$
P R\left(\hat{\delta}_{G Q}\right)=E_{\pi}\left(\tau(\lambda)(\lambda-\delta)^{2}\right)
$$

The Entropy loss function is defined as $L(\lambda, \delta)=\left(\frac{\delta}{\lambda}\right)^{p}-p \log \left(\frac{\delta}{\lambda}\right)-1$. The Bayesian estimator of $\lambda$ is

$$
\hat{\delta}_{E}=E_{\pi}\left(\lambda^{-p}\right)^{\frac{-1}{p}}
$$

where $p$ is a selected constant and the associated posterior risk is

$$
\operatorname{PR}\left(\hat{\delta}_{E}\right)=p\left[E_{\pi}\left(\log \left(\lambda-\log \left(\hat{\delta}_{E}\right)\right)\right)\right]
$$

The Linex loss function is defined as $L(\lambda, \delta)=\exp (r(\delta-\lambda))-r(\delta-\lambda)-1$. The Bayesian estimator of $\lambda$ is

$$
\hat{\delta}_{L}=\frac{-1}{r} \log \left(E_{\pi}(\exp (-r \lambda))\right)
$$

where $r$ is a selected constant and the corresponding the posterior risk is

$$
P R\left(\hat{\delta}_{L}\right)=r\left(\hat{\delta}_{G Q}-\hat{\delta}_{L}\right)
$$

(iii) Bayesian Estimators and their posterior risks.

In the case of the generalized quadratic loss function, the Bayes estimators are given by the formulas:

$$
\begin{array}{r}
\hat{\theta}_{G Q}=\frac{\iint_{0}^{+\infty} B^{-n} C^{n-m} \theta^{b+3 m+\alpha-1} e^{(-a \theta-c \beta+m \beta \theta)} \prod_{i=1}^{m} x_{i}\left(1+x_{i}\right) e^{\theta x_{i}} \mathrm{~d} \theta \mathrm{d} \beta}{\iint_{0}^{+\infty} B^{-n} C^{n-m} \theta^{b+3 m+\alpha-2} e^{(-a \theta-c \beta+m \beta \theta)} \prod_{i=1}^{m} x_{i}\left(1+x_{i}\right) e^{\theta x_{i}} \mathrm{~d} \theta \mathrm{d} \beta}, \\
\hat{\beta}_{G Q}=\frac{\iint_{0}^{+\infty} B^{-n} C^{n-m} \theta^{b_{1}-1+3 m+} \beta^{\alpha} e^{\left(-a_{1} \theta-a_{2} \beta+m \beta \theta\right)} \prod_{i=1}^{m} x_{i}\left(1+x_{i}\right) e^{\theta x_{i}} \mathrm{~d} \theta \mathrm{d} \beta}{\iint_{0}^{+\infty} B^{-n} C^{n-m} \theta^{b_{1}-1+3 m} \beta^{\alpha-1} e^{\left(-a_{1} \theta-a_{2} \beta+m \beta \theta\right)} \prod_{i=1}^{m} x_{i}\left(1+x_{i}\right) e^{\theta x_{i}} \mathrm{~d} \theta \mathrm{d} \beta} .
\end{array}
$$

The corresponding posterior risks are then

$$
P R\left(\hat{\theta}_{G Q}\right)=E_{\pi}\left(\theta^{\alpha+1}\right)-2 \hat{\theta}_{G Q} E_{\pi}\left(\theta^{\alpha}\right)+\hat{\theta}_{G Q}^{2} E_{\pi}\left(\theta^{\alpha-1}\right)
$$


and

$$
P R\left(\hat{\beta}_{G Q}\right)=E_{\pi}\left(\beta^{\alpha+1}\right)-2 \hat{\beta}_{G Q} E_{\pi}\left(\beta^{\alpha}\right)+\hat{\beta}_{G Q}^{2} E_{\pi}\left(\beta^{\alpha-1}\right) .
$$

Under the entropy loss function, we obtain the following estimators:

$$
\begin{gathered}
\hat{\theta}_{E}=\left[K \iint_{0}^{+\infty} B^{-n} C^{n-m} \theta^{b-1+3 m-p} e^{(-a \theta-c \beta+m \beta \theta)} \prod_{i=1}^{m} x_{i}\left(1+x_{i}\right) e^{\theta x_{i}} \mathrm{~d} \theta \mathrm{d} \beta\right]^{-\frac{1}{p}}, \\
\hat{\beta}_{E}=\left[K \iint_{0}^{+\infty} B^{-n} C^{n-m} \theta^{b-1+3 m} \beta^{-p} e^{(-a \theta-c \beta+m \beta \theta)} \prod_{i=1}^{m} x_{i}\left(1+x_{i}\right) e^{\theta x_{i}} \mathrm{~d} \theta \mathrm{d} \beta\right]^{-\frac{1}{p}} .
\end{gathered}
$$

The corresponding posterior risks are then

$$
\begin{gathered}
P R\left(\hat{\theta}_{E}\right)=P E_{\pi}\left(\log (\theta)-\log \left(\hat{\theta}_{E}\right)\right), \\
P R\left(\hat{\beta}_{E}\right)=P E_{\pi}\left(\log (\beta)-\log \left(\hat{\beta}_{E}\right)\right) .
\end{gathered}
$$

Under the Linex loss function, we obtain the following estimators:

$$
\begin{aligned}
& \hat{\theta}_{L}=\frac{K}{r} \ln \left[\iint_{0}^{+\infty} B^{-n} C^{n-m} \theta^{b-1+3 m} e^{(-a \theta-c \beta+m \beta \theta-r \theta)} \prod_{i=1}^{m} x_{i}\left(1+x_{i}\right) e^{\theta x_{i}} \mathrm{~d} \theta \mathrm{d} \beta\right], \\
& \hat{\beta}_{L}=\frac{K}{r} \ln \left[\iint_{0}^{+\infty} B^{-n} C^{n-m} \theta^{b-1+3 m} e^{(-a \theta-c \beta+m \beta \theta-r \beta)} \prod_{i=1}^{m} x_{i}\left(1+x_{i}\right) e^{\theta x_{i}} \mathrm{~d} \theta \mathrm{d} \beta\right],
\end{aligned}
$$

and he corresponding posterior risks are

$$
\begin{aligned}
& P R\left(\hat{\theta}_{L}\right)=r\left(\hat{\theta}_{G Q}-\hat{\theta}_{L}\right), \\
& \operatorname{PR}\left(\hat{\beta}_{L}\right)=r\left(\hat{\beta}_{G Q}-\hat{\beta}_{L}\right) .
\end{aligned}
$$

In the next section, we will use an MCMC method to evaluate these estimators.

\section{Simulation study}

In this section, we present some simulation results to compare the performance of the different estimations that are proposed in this paper. We compare the performance of the ML and the Bayes estimators of the unknown parameters for the UTZ distribution under type II censored data.

For given hyper-parameters $a=b=c=d=1$, and $\theta=1, \beta=1,5$, using $N=5000$ samples from the UTZ distribution, we obtain the following results.

\subsection{Maximum Likelihood estimators}

We have used the R package $B B$ to derive the numerical values of the ML estimators: From Table 1, we see that the estimated values of $\theta$ are close to its true value. Moreover, the quadratic error is small. However, the estimated values of $\beta$ are not close to its true value.

\subsection{Bayesian estimators}

To evaluate the Bayesian estimators, we will use the Metropolis-Hastings algorithm. In the case of the generalized quadratic loss function we use $\alpha \in\{2,-1.5,-1,-0.5,0.5,1,1.5,2\}$, and for the Entropy loss function we use $p \in\{2,-1.5,-1,-0.5,0.5,1,1.5,2\}$. Finally, for the Linex loss function we use $r \in$ $\{2,-1.5,-1,-0.5,0.5,1,1.5,2\}$. 
Table 1: The ML estimators of the parameters with quadratic error (in brackets).

\begin{tabular}{|c|c|c|c|}
\hline$n$ & $m$ & parameter & MLE \\
\hline \multirow[t]{2}{*}{10} & \multirow{2}{*}{8} & $\theta$ & $0.7742(0.0735)$ \\
\hline & & $\beta$ & $1.9654 \quad(0.1763)$ \\
\hline \multirow[t]{2}{*}{30} & \multirow{2}{*}{24} & $\theta$ & $0.9135 \quad(0.0078)$ \\
\hline & & $\beta$ & $1.6302(0.0152)$ \\
\hline \multirow[t]{2}{*}{50} & \multirow{2}{*}{40} & $\theta$ & $0.9573(0.0045)$ \\
\hline & & $\beta$ & $1.6873(0.0078)$ \\
\hline \multirow[t]{2}{*}{100} & \multirow{2}{*}{80} & $\theta$ & $0.9843(0.0075)$ \\
\hline & & $\beta$ & $1.5259(0.0005)$ \\
\hline \multirow[t]{2}{*}{200} & \multirow{2}{*}{160} & $\theta$ & $0.9397(0.0054)$ \\
\hline & & $\beta$ & $1.6434(0.0217)$ \\
\hline
\end{tabular}

In Table 2 below we display the values of the Bayesian estimators and their corresponding posterior risks, in brackets, under the generalized quadratic loss function.

Table 2: Bayes estimators and $P R$ (in brackets) under generalized quadratic loss function.

\begin{tabular}{|c|c|c|c|c|c|c|c|c|c|c|}
\hline \multirow{2}{*}{$N$} & \multirow{2}{*}{$m$} & \multirow{2}{*}{ Parameter } & \multicolumn{8}{|c|}{$\alpha$} \\
\hline & & & -2 & $-1,5$ & -1 & $-0,5$ & 0,5 & 1 & 1,5 & 2 \\
\hline \multirow{2}{*}{10} & \multirow{2}{*}{8} & $\theta$ & $\begin{array}{c}1,0881 \\
(0,0065)\end{array}$ & $\begin{array}{c}1,0925 \\
(0,0079)\end{array}$ & $\begin{array}{c}1,0990 \\
(0,0087)\end{array}$ & $\begin{array}{c}1,0994 \\
(0,0089)\end{array}$ & $\begin{array}{l}1,11109 \\
(0,0095)\end{array}$ & $\begin{array}{c}1,1175 \\
(0,0091)\end{array}$ & $\begin{array}{c}1,1099 \\
(0,0098)\end{array}$ & $\begin{array}{c}1,1241 \\
(0,01193)\end{array}$ \\
\hline & & $\beta$ & $\begin{array}{c}1,1941 \\
(0,0432)\end{array}$ & $\begin{array}{c}1,2191 \\
(0,0593)\end{array}$ & $\begin{array}{c}1,2657 \\
(0,7091)\end{array}$ & $\begin{array}{c}1,2999 \\
(0,0825)\end{array}$ & $\begin{array}{c}1,3891 \\
(0,0909)\end{array}$ & $\begin{array}{c}1,4228 \\
(0,1094)\end{array}$ & $\begin{array}{c}1,4768 \\
(0,1241)\end{array}$ & $\begin{array}{c}1,5198 \\
(0,1451)\end{array}$ \\
\hline \multirow{2}{*}{30} & \multirow[b]{2}{*}{24} & $\theta$ & $\begin{array}{c}1,0739 \\
(0,0043)\end{array}$ & $\begin{array}{c}1,0829 \\
(0,0058)\end{array}$ & $\begin{array}{c}1,0799 \\
(0,0066)\end{array}$ & $\begin{array}{c}1,0884 \\
(0,0061)\end{array}$ & $\begin{array}{c}1,0957 \\
(0,0061)\end{array}$ & $\begin{array}{c}1,0965 \\
(0,0069)\end{array}$ & $\begin{array}{c}1,1021 \\
(0,0077)\end{array}$ & $\begin{array}{c}1,0971 \\
(0,0089)\end{array}$ \\
\hline & & $\beta$ & $\begin{array}{c}1,1805 \\
(0,0429)\end{array}$ & $\begin{array}{c}1,2201 \\
(0,0509)\end{array}$ & $\begin{array}{c}1,2297 \\
(0,0671)\end{array}$ & $\begin{array}{c}1,3011 \\
(0,0711)\end{array}$ & $\begin{array}{c}1,3915 \\
(0,0802)\end{array}$ & $\begin{array}{c}1,4135 \\
(0,0999)\end{array}$ & $\begin{array}{c}1,4841 \\
(0,1097)\end{array}$ & $\begin{array}{c}1,4955 \\
(0,1228)\end{array}$ \\
\hline \multirow{2}{*}{50} & \multirow{2}{*}{40} & $\theta$ & $\begin{array}{c}1,0739 \\
(0,0051)\end{array}$ & $\begin{array}{c}1,0792 \\
(0,0058)\end{array}$ & $\begin{array}{c}1,0825 \\
(0,0061)\end{array}$ & $\begin{array}{c}1,0888 \\
(0,0070)\end{array}$ & $\begin{array}{c}1,0922 \\
(0,0077)\end{array}$ & $\begin{array}{c}1,0977 \\
(0,0078)\end{array}$ & $\begin{array}{c}1,0990 \\
(0,0081)\end{array}$ & $\begin{array}{c}1,1035 \\
(0,0085) \\
\end{array}$ \\
\hline & & $\beta$ & $\begin{array}{c}1,1670 \\
(0,0439)\end{array}$ & $\begin{array}{c}1,1799 \\
(0,0499)\end{array}$ & $\begin{array}{c}1,2039 \\
(0,0633)\end{array}$ & $\begin{array}{l}1,2701 \\
(0,711) \\
\end{array}$ & $\begin{array}{l}1,3591 \\
(0,995) \\
\end{array}$ & $\begin{array}{c}1,3803 \\
(0,1071)\end{array}$ & $\begin{array}{c}1,4191 \\
(0,1181)\end{array}$ & $\begin{array}{c}0,4702 \\
(0,1201)\end{array}$ \\
\hline \multirow{2}{*}{100} & \multirow{2}{*}{80} & $\theta$ & $\begin{array}{c}1,2111 \\
(0,0016)\end{array}$ & $\begin{array}{c}0,2132 \\
(0,0018)\end{array}$ & $\begin{array}{c}0,2135 \\
(0,0019)\end{array}$ & $\begin{array}{c}0,2147 \\
(0,0021)\end{array}$ & $\begin{array}{c}0,2151 \\
(0,0026)\end{array}$ & $\begin{array}{c}0,2159 \\
(0,0028)\end{array}$ & $\begin{array}{c}0,2701 \\
() 0,0030\end{array}$ & $\begin{array}{c}0,2209 \\
(0,0030)\end{array}$ \\
\hline & & $\beta$ & $\begin{array}{c}1,7218 \\
(0,0006)\end{array}$ & $\begin{array}{c}1,7220 \\
(0,0007)\end{array}$ & $\begin{array}{c}1,7234 \\
(0,0008)\end{array}$ & $\begin{array}{c}1,7245 \\
(0,0011)\end{array}$ & $\begin{array}{c}1,7258 \\
(0,0023)\end{array}$ & $\begin{array}{c}1,7268 \\
(0,0028)\end{array}$ & $\begin{array}{c}1,7301 \\
(0,0035)\end{array}$ & $\begin{array}{c}1,7290 \\
(0,0048)\end{array}$ \\
\hline \multirow{2}{*}{200} & \multirow{2}{*}{160} & $\theta$ & $\begin{array}{c}1,2108 \\
(0,0013)\end{array}$ & $\begin{array}{c}1,2118 \\
(0,0015)\end{array}$ & $\begin{array}{c}1,2127 \\
(0,0016)\end{array}$ & $\begin{array}{c}1,2138 \\
(0,0018)\end{array}$ & $\begin{array}{c}2,1839 \\
(0,0020) \\
\end{array}$ & $\begin{array}{c}2,1841 \\
(0,0031)\end{array}$ & $\begin{array}{c}2,1841 \\
(0,0042) \\
\end{array}$ & $\begin{array}{c}2,1851 \\
(0,0066) \\
\end{array}$ \\
\hline & & $\beta$ & $\begin{array}{c}1,7105 \\
(0,0005)\end{array}$ & $\begin{array}{c}1,7115 \\
(0,0007)\end{array}$ & $\begin{array}{c}1,7120 \\
(0,0008)\end{array}$ & $\begin{array}{c}1,7131 \\
(0,0012)\end{array}$ & $\begin{array}{c}1,7139 \\
(0,0019)\end{array}$ & $\begin{array}{c}1,7149 \\
(0,0025)\end{array}$ & $\begin{array}{c}1,7158 \\
(0,0033) \\
\end{array}$ & $\begin{array}{c}1,7169 \\
(0,0046) \\
\end{array}$ \\
\hline
\end{tabular}

We note that the chosen value $\alpha=-2$ provides the best posterior risks, which presents the best estimator for the generalised quadratic loss function case. Also, when $n$ is large, we obtain the smallest posterior risks.

Table 3 presents the values of the Bayesian estimators and their corresponding posterior risks, in brackets, under the Entropy loss function.

From that table, the value $p=-0.5$ provides the best posterior risks, which presents the best estimations for the Entropy loss function case. Also, when $n=100$ and $n=200$, we obtain the smallest posterior risks. Table 4 presents the values of the Bayesian estimators and their corresponding posterior risks, in brackets, under the Linex loss function. 
Table 3: Bayes estimators and $P R$ (in brackets) under entropy loss function.

\begin{tabular}{|c|c|c|c|c|c|c|c|c|c|c|}
\hline \multirow{2}{*}{$n$} & \multirow{2}{*}{$m$} & \multirow{2}{*}{ Parameter } & \multicolumn{8}{|c|}{$p$} \\
\hline & & & -2 & $-1,5$ & -1 & $-0,5$ & 0,5 & 1 & 1,5 & 2 \\
\hline \multirow[b]{2}{*}{10} & \multirow{2}{*}{8} & $\theta$ & $\begin{array}{c}1,1097 \\
(0,0099)\end{array}$ & $\begin{array}{c}1,1067 \\
(0,0091)\end{array}$ & $\begin{array}{c}1,1053 \\
(0,0035)\end{array}$ & $\begin{array}{c}1,1041 \\
(0,0009)\end{array}$ & $\begin{array}{c}1,0995 \\
(0,0008)\end{array}$ & $\begin{array}{c}0,0981 \\
(0,0038)\end{array}$ & $\begin{array}{l}0,0942 \\
(0,008)\end{array}$ & $\begin{array}{c}0,0911 \\
(0,0155)\end{array}$ \\
\hline & & $\beta$ & $\begin{array}{c}1,4579 \\
(0,0997)\end{array}$ & $\begin{array}{c}1,4407 \\
(0,0611)\end{array}$ & $\begin{array}{c}1,4239 \\
(0,0199)\end{array}$ & $\begin{array}{c}1,4177 \\
(00,0072\end{array}$ & $\begin{array}{c}1,3639 \\
(0,0071)\end{array}$ & $\begin{array}{c}1,3491 \\
(0,0308)\end{array}$ & $\begin{array}{c}1,3188 \\
(0,0699)\end{array}$ & $\begin{array}{c}1,2909 \\
(0,1249)\end{array}$ \\
\hline \multirow[b]{2}{*}{30} & \multirow[b]{2}{*}{24} & $\theta$ & $\begin{array}{c}1,0999 \\
(0,0191)\end{array}$ & $\begin{array}{c}1,0981 \\
(0,0065)\end{array}$ & $\begin{array}{c}1,0898 \\
(0,0042)\end{array}$ & $\begin{array}{c}1,0863 \\
(0,0007)\end{array}$ & $\begin{array}{c}1,0831 \\
(0,0008)\end{array}$ & $\begin{array}{l}0,0817 \\
(0,003)\end{array}$ & $\begin{array}{l}1,0799 \\
(0,009)\end{array}$ & $\begin{array}{c}1,0781 \\
(0,0111)\end{array}$ \\
\hline & & $\beta$ & $\begin{array}{c}1,4325 \\
(0,1011)\end{array}$ & $\begin{array}{c}1,4151 \\
(0,0622)\end{array}$ & $\begin{array}{c}1,3966 \\
(0,0281)\end{array}$ & $\begin{array}{c}1,3709 \\
(0,0074)\end{array}$ & $\begin{array}{c}1,3061 \\
(0,0077)\end{array}$ & $\begin{array}{c}1,3185 \\
(0,0319)\end{array}$ & $\begin{array}{c}1,2908 \\
(0,0690)\end{array}$ & $\begin{array}{c}1,2901 \\
(0,1246)\end{array}$ \\
\hline \multirow{2}{*}{50} & \multirow{2}{*}{40} & $\theta$ & $\begin{array}{c}1,7644 \\
(0,1173)\end{array}$ & $\begin{array}{c}1,7188 \\
(0,1443)\end{array}$ & $\begin{array}{c}1,6701 \\
(0,0667)\end{array}$ & $\begin{array}{c}1,6205 \\
(0,0171)\end{array}$ & $\begin{array}{c}1,5809 \\
(0,0729)\end{array}$ & $\begin{array}{c}1,4830 \\
(0,0733)\end{array}$ & $\begin{array}{c}1,3990 \\
(0,1644)\end{array}$ & $\begin{array}{c}1,3458 \\
(0,2849)\end{array}$ \\
\hline & & $\beta$ & $\begin{array}{c}1,4259 \\
(0,0944)\end{array}$ & $\begin{array}{c}1,4077 \\
(0,0661)\end{array}$ & $\begin{array}{c}1,3881 \\
(0,0303)\end{array}$ & $\begin{array}{c}1,3633 \\
(0,0073)\end{array}$ & $\begin{array}{c}1,3255 \\
(0,0065)\end{array}$ & $\begin{array}{l}1,3055 \\
(0,319)\end{array}$ & $\begin{array}{l}1,2839 \\
(0,009)\end{array}$ & $\begin{array}{c}1,2588 \\
(0,1265)\end{array}$ \\
\hline \multirow{2}{*}{100} & \multirow{2}{*}{80} & $\theta$ & $\begin{array}{c}1,2101 \\
(0,0038)\end{array}$ & $\begin{array}{c}1,2096 \\
(0,0022)\end{array}$ & $\begin{array}{c}1,2089 \\
(0,0009)\end{array}$ & $\begin{array}{c}1,2084 \\
(0,0002)\end{array}$ & $\begin{array}{c}1,2073 \\
(0,0003)\end{array}$ & $\begin{array}{c}1,2067 \\
(0,0009)\end{array}$ & $\begin{array}{c}1,2061 \\
(0,0032)\end{array}$ & $\begin{array}{c}1,2056 \\
(0,0038)\end{array}$ \\
\hline & & $\beta$ & $\begin{array}{c}1,7078 \\
(0,0018)\end{array}$ & $\begin{array}{c}1,7071 \\
(0,0010)\end{array}$ & $\begin{array}{c}1,7060 \\
(0,0005) \\
\end{array}$ & $\begin{array}{c}1,7062 \\
(0,0001) \\
\end{array}$ & $\begin{array}{c}1,7054 \\
(0,0001) \\
\end{array}$ & $\begin{array}{c}1,7049 \\
(0,0006) \\
\end{array}$ & $\begin{array}{c}1,7046 \\
(0,0010) \\
\end{array}$ & $\begin{array}{c}1,7042 \\
(0,0021) \\
\end{array}$ \\
\hline \multirow{2}{*}{200} & \multirow{2}{*}{160} & $\theta$ & $\begin{array}{c}1,2188 \\
(0,0031)\end{array}$ & $\begin{array}{c}1,2179 \\
(0,0017)\end{array}$ & $\begin{array}{c}1,2169 \\
(0,0009)\end{array}$ & $\begin{array}{c}1,2167 \\
(0,0001)\end{array}$ & $\begin{array}{c}1,2154 \\
(0,0001)\end{array}$ & $\begin{array}{c}1,2148 \\
(0,0009)\end{array}$ & $\begin{array}{c}1,2144 \\
(0,0019)\end{array}$ & $\begin{array}{c}1,2139 \\
(0,0032)\end{array}$ \\
\hline & & $\beta$ & $\begin{array}{c}1,7071 \\
(0,0018)\end{array}$ & $\begin{array}{c}1,7060 \\
(0,0010)\end{array}$ & $\begin{array}{c}0,7059 \\
(0,0006)\end{array}$ & $\begin{array}{c}1,7051 \\
(0,0002)\end{array}$ & $\begin{array}{c}1,7044 \\
(0,0001)\end{array}$ & $\begin{array}{c}1,7037 \\
(0,0005)\end{array}$ & $\begin{array}{l}1,7034 \\
(0,014) \\
\end{array}$ & $\begin{array}{c}1,7030 \\
(0,0019) \\
\end{array}$ \\
\hline
\end{tabular}

Table 4: Bayes estimators and $P R$ (in brackets) under Linex loss function.

\begin{tabular}{|c|c|c|c|c|c|c|c|c|c|c|}
\hline \multirow{2}{*}{$N$} & \multirow{2}{*}{$m$} & \multirow{2}{*}{ Parameter } & \multicolumn{8}{|c|}{$r$} \\
\hline & & & -2 & $-1,5$ & -1 & $-0,5$ & 0,5 & 1 & 1,5 & 2 \\
\hline \multirow{2}{*}{10} & \multirow{2}{*}{8} & $\theta$ & $\begin{array}{c}1,1251 \\
(0,0195)\end{array}$ & $\begin{array}{c}1,1228 \\
(0,0105)\end{array}$ & $\begin{array}{c}1,1201 \\
(0,0039)\end{array}$ & $\begin{array}{c}1,1189 \\
(0,0011)\end{array}$ & $\begin{array}{c}1,1132 \\
(0,0012)\end{array}$ & $\begin{array}{c}1,1108 \\
(0,0049)\end{array}$ & $\begin{array}{c}1,1082 \\
(0,0107)\end{array}$ & $\begin{array}{c}1,1052 \\
(0,0185)\end{array}$ \\
\hline & & $\beta$ & $\begin{array}{c}1,5309 \\
(0,1666)\end{array}$ & $\begin{array}{c}1,5117 \\
(0,1033)\end{array}$ & $\begin{array}{c}1,4806 \\
(0,0411)\end{array}$ & $\begin{array}{c}1,4492 \\
(0,0177)\end{array}$ & $\begin{array}{c}1,4132 \\
(0,0167)\end{array}$ & $\begin{array}{c}1,3581 \\
(0,0433)\end{array}$ & $\begin{array}{c}0,3495 \\
(0,01213)\end{array}$ & $\begin{array}{c}0,3345 \\
(0,03131)\end{array}$ \\
\hline \multirow{2}{*}{30} & \multirow{2}{*}{24} & $\theta$ & $\begin{array}{c}1,1058 \\
(0,0147) \\
\end{array}$ & $\begin{array}{c}1,1041 \\
(0,0085)\end{array}$ & $\begin{array}{c}1,1022 \\
(0,0039) \\
\end{array}$ & $\begin{array}{c}1,1005 \\
(0,0007) \\
\end{array}$ & $\begin{array}{c}1,0991 \\
(0,0007) \\
\end{array}$ & $\begin{array}{c}1,0948 \\
(0,0038) \\
\end{array}$ & $\begin{array}{c}1,0930 \\
(0,3577) \\
\end{array}$ & $\begin{array}{c}1,0912 \\
(0,6107) \\
\end{array}$ \\
\hline & & $\beta$ & $\begin{array}{c}1,4951 \\
(0,1678)\end{array}$ & $\begin{array}{c}1,4759 \\
(0,1001)\end{array}$ & $\begin{array}{c}1,4547 \\
(0,1041)\end{array}$ & $\begin{array}{c}1,4315 \\
(0,0481)\end{array}$ & $\begin{array}{c}1,3815 \\
(0,0183)\end{array}$ & $\begin{array}{c}1,3538 \\
(0,0131)\end{array}$ & $\begin{array}{c}1,3272 \\
(0,0119)\end{array}$ & $\begin{array}{c}1,3014 \\
(0,1205)\end{array}$ \\
\hline \multirow{2}{*}{50} & \multirow{2}{*}{40} & $\theta$ & $\begin{array}{c}1,0939 \\
(0,0151)\end{array}$ & $\begin{array}{c}1,0919 \\
(0,0081)\end{array}$ & $\begin{array}{c}1,0815 \\
(0,0038)\end{array}$ & $\begin{array}{c}1,0861 \\
(0,0009)\end{array}$ & $\begin{array}{c}1,0813 \\
(0,0007)\end{array}$ & $\begin{array}{c}1,0811 \\
(0,0038)\end{array}$ & $\begin{array}{c}1,0799 \\
(0,0081)\end{array}$ & $\begin{array}{c}1,0771 \\
(0,0111)\end{array}$ \\
\hline & & $\beta$ & $\begin{array}{c}1,4821 \\
(0,1884)\end{array}$ & $\begin{array}{c}1,4639 \\
(0,0581)\end{array}$ & $\begin{array}{c}1,4455 \\
(0,0519)\end{array}$ & $\begin{array}{c}1,4193 \\
(0,0131)\end{array}$ & $\begin{array}{c}1,3609 \\
(0,0199)\end{array}$ & $\begin{array}{c}1,3338 \\
(0,0655)\end{array}$ & $\begin{array}{c}1,3160 \\
(0,1155)\end{array}$ & $\begin{array}{c}1,2915 \\
(0,2109)\end{array}$ \\
\hline \multirow{2}{*}{100} & \multirow{2}{*}{80} & $\theta$ & $\begin{array}{c}2,2070 \\
(0,0057)\end{array}$ & $\begin{array}{c}2,2061 \\
(0,0015)\end{array}$ & $\begin{array}{c}2,2041 \\
(0,0013)\end{array}$ & $\begin{array}{c}2,2039 \\
(0,0003)\end{array}$ & $\begin{array}{c}2,0019 \\
(0,0004)\end{array}$ & $\begin{array}{c}2,2017 \\
(0,0015)\end{array}$ & $\begin{array}{c}2,0011 \\
(0,0032)\end{array}$ & $\begin{array}{c}2,0915 \\
(0,0056)\end{array}$ \\
\hline & & $\beta$ & $\begin{array}{c}1,7094 \\
(0,0057)\end{array}$ & $\begin{array}{c}1,7091 \\
(0,0032)\end{array}$ & $\begin{array}{c}1,7080 \\
(0,0014)\end{array}$ & $\begin{array}{c}1,7077 \\
(0,0004)\end{array}$ & $\begin{array}{c}1,7059 \\
(0,0013)\end{array}$ & $\begin{array}{c}1,7055 \\
(0,0049)\end{array}$ & $\begin{array}{c}1,7044 \\
(0,0031)\end{array}$ & $\begin{array}{c}1,7038 \\
(0,0054)\end{array}$ \\
\hline \multirow{2}{*}{200} & \multirow{2}{*}{160} & $\theta$ & $\begin{array}{c}1,2191 \\
(0,0049)\end{array}$ & $\begin{array}{c}1,2181 \\
(0,0027)\end{array}$ & $\begin{array}{c}1,2179 \\
(0,0012)\end{array}$ & $\begin{array}{c}1,2174 \\
(0,0003)\end{array}$ & $\begin{array}{c}1,2153 \\
(0,0003)\end{array}$ & $\begin{array}{c}1,2151 \\
(0,0012)\end{array}$ & $\begin{array}{c}1,2133 \\
(0,0025)\end{array}$ & $\begin{array}{c}1,2129 \\
(0,0049)\end{array}$ \\
\hline & & $\beta$ & $\begin{array}{c}1,7031 \\
(0,0051)\end{array}$ & $\begin{array}{c}1,7059 \\
(0,0031)\end{array}$ & $\begin{array}{c}1,7054 \\
(0,0013)\end{array}$ & $\begin{array}{c}1,7045 \\
(0,0004)\end{array}$ & $\begin{array}{c}1,7011 \\
(0,0004)\end{array}$ & $\begin{array}{c}1,7041 \\
(0,0012)\end{array}$ & $\begin{array}{c}1,7039 \\
(0,0027)\end{array}$ & $\begin{array}{c}1,7015 \\
(0,0041)\end{array}$ \\
\hline
\end{tabular}

Here, the value $r=-0.5$ provides the best posterior risks, which presents the best estimations for the Linex loss function case. Also, when $n$ is high, we obtain the smallest posterior risks.

In Table 5, we display the three loss functions corresponding to the best results, i.e. when $\alpha=-2, p=-0.5$ and $r=-0.5$. 
Table 5: Bays estimators and PR (in brackets) under the three loss function.

\begin{tabular}{|c|c|c|c|c|c|}
\hline$n$ & $m$ & Parameter & $G Q(\alpha=-2)$ & $\begin{array}{c}\text { Entropy }(P= \\
-0,5)\end{array}$ & $\begin{array}{c}\text { Linex } \\
(r=-0,5)\end{array}$ \\
\hline \multirow[b]{2}{*}{10} & \multirow[b]{2}{*}{8} & $\theta$ & $\begin{array}{c}1,0881 \\
(0,0065)\end{array}$ & $\begin{array}{c}1,1041 \\
(0,0009)\end{array}$ & $\begin{array}{c}1,1189 \\
(0,0011)\end{array}$ \\
\hline & & $\beta$ & $\begin{array}{c}1,1941 \\
(0,0432)\end{array}$ & $\begin{array}{c}1,4177 \\
(0,0072)\end{array}$ & $\begin{array}{c}1,4492 \\
(0,0177)\end{array}$ \\
\hline \multirow[b]{2}{*}{30} & \multirow[b]{2}{*}{24} & $\theta$ & $\begin{array}{c}1,0739 \\
(0,0043)\end{array}$ & $\begin{array}{c}1,0863 \\
(0,0007)\end{array}$ & $\begin{array}{c}1,1005 \\
(0,0007)\end{array}$ \\
\hline & & $\beta$ & $\begin{array}{c}1,1805 \\
(0,0429)\end{array}$ & $\begin{array}{c}1,3709 \\
(0,0074)\end{array}$ & $\begin{array}{c}1,4315 \\
(0,0481)\end{array}$ \\
\hline \multirow{2}{*}{50} & \multirow{2}{*}{40} & $\theta$ & $\begin{array}{c}1,0739 \\
(0,0051)\end{array}$ & $\begin{array}{c}1,6205 \\
(0,0171)\end{array}$ & $\begin{array}{c}1,0861 \\
(0,0009)\end{array}$ \\
\hline & & $\beta$ & $\begin{array}{c}1,1670 \\
(0,0439)\end{array}$ & $\begin{array}{c}1,3633 \\
(0,0073)\end{array}$ & $\begin{array}{c}1,4193 \\
(0,0131)\end{array}$ \\
\hline \multirow{2}{*}{100} & \multirow{2}{*}{80} & $\theta$ & $\begin{array}{c}1,2111 \\
(0,0016)\end{array}$ & $\begin{array}{c}1,2084 \\
(0,0002)\end{array}$ & $\begin{array}{c}2,2039 \\
(0,0003)\end{array}$ \\
\hline & & $\beta$ & $\begin{array}{c}1,7218 \\
(0,0006)\end{array}$ & $\begin{array}{c}1,7062 \\
(0,0001)\end{array}$ & $\begin{array}{c}1,7077 \\
(0,0004)\end{array}$ \\
\hline \multirow{2}{*}{200} & \multirow{2}{*}{160} & $\theta$ & $\begin{array}{c}1,2108 \\
(0,0013)\end{array}$ & $\begin{array}{c}1,2167 \\
(0,0001)\end{array}$ & $\begin{array}{c}1,2174 \\
(0,0003)\end{array}$ \\
\hline & & $\beta$ & $\begin{array}{c}1,7105 \\
(0,0005)\end{array}$ & $\begin{array}{c}1,7051 \\
(0,0002)\end{array}$ & $\begin{array}{c}1,7045 \\
(0,0004)\end{array}$ \\
\hline
\end{tabular}

Summing up, from the displayed values, it follows that the best results are obtained by the Entropy loss function.

\subsection{Comparison of the estimators using Pitman's closeness criterion}

Definition 4.1. An estimator $\theta_{1}$ of a parameter $\theta$ dominates another estimator $\theta_{2}$ in the sense of Pitman's closeness criterion if, for all $\theta \in \Theta$,

$$
P_{\theta}\left[\left|\theta_{1}-\theta\right|<\left|\theta_{2}-\theta\right|\right]>0.5
$$

In the following table, we present the values of the Pitman probabilities which allow us to compare the Bayesian estimators with the ML estimators under the three loss functions when $\alpha=-2 . p=-0.5 . r=-0.5$. if the probability is greater than 0.5 , the Bayesian estimators are better than the ML estimators.

Table 6: Pitman comparison of the estimators of $\theta$ and $\beta$.

\begin{tabular}{|c|c|c|c|c|c|}
\hline$n$ & $m$ & Parameter & $G Q(\alpha=-2)$ & $\begin{array}{c}\text { Entropy }(p= \\
-0,5)\end{array}$ & $\begin{array}{c}\text { Linex } \\
(r=-0,5)\end{array}$ \\
\hline \multirow{2}{*}{10} & \multirow{2}{*}{8} & $\theta$ & 0,6791 & 0,635 & 0,659 \\
\hline & & $\beta$ & 0,657 & 0,519 & 0,635 \\
\hline \multirow{2}{*}{30} & \multirow{2}{*}{24} & $\theta$ & 0,579 & 0,558 & 0,569 \\
\hline & & $\beta$ & 0,534 & 0,499 & 0,516 \\
\hline \multirow{2}{*}{50} & \multirow{2}{*}{40} & $\theta$ & 0,539 & 0,519 & 0,600 \\
\hline & & $\beta$ & 0,441 & 0,385 & 0,381 \\
\hline \multirow{2}{*}{100} & \multirow{2}{*}{80} & $\theta$ & 0,130 & 0,1327 & 0,1809 \\
\hline & & $\beta$ & 0,491 & 0,335 & 0,394 \\
\hline \multirow{2}{*}{200} & \multirow{2}{*}{160} & $\theta$ & 0,2214 & 0,071 & 0,091 \\
\hline & & $\beta$ & 0,288 & 0,249 & 0,2191 \\
\hline
\end{tabular}


According to Pitman's criterion, the Bayesian estimators of $\theta$ are better than $\theta_{M L E}$ when $n$ is small. Also the generalized quadratic loss function has the best values in comparison with the other two loss functions. However, $\beta_{M L E}$ is closer to the true value than all the Bayesian estimators. Moreover, $\theta_{M L E}$ and $\beta_{M L E}$ perform better than the corresponding Bayesian estimators, when $n$ is large.

Definition 4.2. The integrated mean square error is defined as

$$
I M S E=\frac{1}{N} \sum_{i=1}^{N}\left(\theta_{i}-\theta\right)^{2} .
$$

In Table 7, below, we display the values of the integrated mean square error of the estimators under the three loss functions and the ML estimators.

Table 7: The IMSE of the estimators of $\theta$ and $\beta$.

\begin{tabular}{|c|c|c|c|c|c|c|}
\hline \multirow{2}{*}{$n$} & \multirow{2}{*}{$m$} & Parameter & MLE & $\begin{array}{c}\text { Generalized } \\
\text { quadratic }\end{array}$ & Entropy & Linex \\
\hline \multirow{2}{*}{10} & \multirow{2}{*}{8} & $\theta$ & 0,2589 & 0,0092 & 0,0224 & 0,0185 \\
\cline { 3 - 7 } & \multirow{2}{*}{24} & $\beta$ & 0,3188 & 0,1065 & 0,1082 & 0,7141 \\
\cline { 3 - 7 } & \multirow{2}{*}{40} & $\beta$ & 0,1045 & 0,0098 & 0,0158 & 0,0149 \\
\hline \multirow{2}{*}{50} & \multirow{2}{*}{10} & $\beta$ & 0,3689 & 0,1159 & 0,1213 & 0,1179 \\
\cline { 3 - 7 } & \multirow{2}{*}{100} & $\beta$ & 0,0801 & 0,0214 & 0,0189 & 0,1494 \\
\hline \multirow{2}{*}{200} & \multirow{2}{*}{160} & $\theta$ & 0,0954 & 0,0259 & 0,0397 & 0,0344 \\
\cline { 3 - 7 } & & $\beta$ & 0,4748 & 0,1214 & 0,1192 & 0,0423 \\
\hline
\end{tabular}

We remark that when $n$ is small the Bayesian estimators of $\theta$ and $\beta$ do provide a small $I M S E$ for the parameters compared with $\theta_{M L E}$ and $\beta_{M L E}$. Also the values provided by the generalized quadratic loss function are relatively close to those provided by the entropy and the Linex loss functions. To conclude, the Bayesian estimators perform better than the ML estimators, and the generalized quadratic loss function gives the smallest IMSE.

\section{Application to real data}

In this section we illustrate the applicability of the UTZ distribution by performing the above estimations using a set of real data. The data set includes the number of gold particles observed on each dystrophin unit (dystrophin is a gene product of possible importance in muscular dystrophies) discussed in Mathews et Appleton (1993) and M Cullen et al. (1990). It is confirmed that the Zeghdoudi distribution fits these data, using the Kolmogorov-Smirnov (K-S) test. The K-S test value is 0.012901 which is smaller than their corresponding critical value at $5 \%$ level of significance, which is 0.025449 (for $n=198$ ). Its P-value is equal to 0.793548 .

The complete observations are displayed in the next table.

\begin{tabular}{|c|c|c|c|c|c|c|}
\hline$x_{i}$ & 1 & 2 & 3 & 4 & 5 & $n$ \\
\hline$n_{i}$ & 122 & 50 & 18 & 4 & 4 & 198 \\
\hline
\end{tabular}

It is clear that the truncation point is 5 in the case of complete data. We assume it 3 for censured data, choosing $m=180$. 
Table 8 presents the Bayesian estimation of the parameters $\theta$ and $\beta$ under the three loss functions with the corresponding posterior risk, and the ML estimators.

Table 8: Likelihood and Bayesian estimation under the three loss functions with posterior risk in brackets.

\begin{tabular}{|c|c|c|c|c|c|c|}
\hline$n$ & $m$ & Parameter & $M L E$ & $G Q(\alpha=-2)$ & $\begin{array}{c}\text { Entropy }(p= \\
-0,5)\end{array}$ & $\begin{array}{c}\text { Linex } \\
(r=-0,5)\end{array}$ \\
\hline \multirow{3}{*}{198} & \multirow{3}{*}{198} & $\theta$ & 0.9807 & $\begin{array}{c}1.2108 \\
(0,0010)\end{array}$ & $\begin{array}{c}1.2018 \\
(0,0003)\end{array}$ & $\begin{array}{c}1.2174 \\
(0,0005)\end{array}$ \\
\cline { 3 - 7 } & & $\beta$ & 1.5987 & $\begin{array}{c}1.6405 \\
(0,0005)\end{array}$ & $\begin{array}{c}1.6051 \\
(0,0009)\end{array}$ & $\begin{array}{c}1.7045 \\
(0,0006)\end{array}$ \\
\hline \multirow{3}{*}{198} & \multirow{2}{*}{180} & $\theta$ & 0.9513 & $\begin{array}{c}1.3408 \\
(0,0017)\end{array}$ & $\begin{array}{c}1.2027 \\
(0,0008)\end{array}$ & $\begin{array}{c}1.2174 \\
(0,0099)\end{array}$ \\
\cline { 3 - 7 } & & $\beta$ & 1.6492 & $\begin{array}{c}1.6605 \\
(0,0008)\end{array}$ & $\begin{array}{c}1.6151 \\
(0,0005)\end{array}$ & $\begin{array}{c}1.7045 \\
(0,0009)\end{array}$ \\
\hline
\end{tabular}

We note that the estimators based on the complete data set provides smaller posterior risk compared with the censored data, which is expected since we lose part of the information using censored data. We also remark the entropy has the smaller posterior risk.

In the next tables, we present the values of the Pitman's criterion using the real data set of the Bayesian estimators and the MLE.

Table 9: Pitman comparison of the estimators of $\theta$ and $\beta$.

\begin{tabular}{|c|c|c|c|c|c|}
\hline$n$ & $m$ & Parameter & $G Q(\alpha=-2)$ & $\begin{array}{c}\text { Entropy }(p= \\
-0,5)\end{array}$ & $\begin{array}{c}\text { Linex } \\
(r=-0,5)\end{array}$ \\
\hline \multirow{2}{*}{198} & \multirow{2}{*}{198} & $\theta$ & 0,213 & 0,1525 & 0,1920 \\
\cline { 3 - 6 } & \multirow{2}{*}{198} & $\beta$ & 0,488 & 0,365 & 0,387 \\
\cline { 3 - 6 } & \multirow{2}{*}{180} & $\theta$ & 0,2334 & 0,141 & 0,099 \\
\cline { 3 - 6 } & & $\beta$ & 0,308 & 0,321 & 0,2219 \\
\hline
\end{tabular}

Comparing the estimators according to Pitman's criterion, the ML estimator performs better than the Bayesian estimator.

Table 9 presents the values of the integrated mean-square error of the Bayesian estimators and the ML estimators.

We note that the values provided by the generalized quadratic loss function are the smallest, and all the Baysian estimators performs better than the ML estimators.

\section{Concluding Remarks}

In this study we proposed a new model, the upper truncated Zeghdoudi (UTZ) distribution. We compared Bayesian estimators of UTZ distribution under various loss functions. The performed Monte-Carlo study showed that the Bayesian approach based on the entropy loss function yielded the best estimator compared to the ones based on the other proposed loss functions. These selected Bayesian estimators are compared with the maximum likelihood estimators of the unknown parameters using Pitman closeness criterion and 
Table 10: IMSE of the estimators of $\theta$ and $\beta$.

\begin{tabular}{|c|c|c|c|c|c|c|}
\hline \multirow{2}{*}{$n$} & $m$ & Parameter & $M L E$ & $G Q(\alpha=-2)$ & $\begin{array}{c}\text { Entropy }(p= \\
-0,5)\end{array}$ & $\begin{array}{c}\text { Linex } \\
(r=-0,5)\end{array}$ \\
\hline \multirow{2}{*}{198} & \multirow{2}{*}{198} & $\theta$ & 0.1893 & 0.1591 & 0.1613 & 0.1603 \\
\cline { 3 - 7 } & \multirow{2}{*}{198} & $\beta$ & 0.5857 & 0.5417 & 0.5793 & 0.5734 \\
\cline { 3 - 7 } & \multirow{2}{*}{180} & $\theta$ & 0.1903 & 0.1093 & 0.1190 & 0.1133 \\
\cline { 3 - 7 } & & $\beta$ & 0.5057 & 0.4350 & 0.5033 & 0.5040 \\
\hline
\end{tabular}

the integrated mean square error, where it showed that when $n$ is small, the Bayesian estimators gave better results, while when $n$ is large enough, the ML estimators are closer to the true values but provide the highest IMSE than the Bayesian estimators. Finally, we show that the same conclusions hold using a set of real data.

In a future work, we plan to construct a mixture of the loss functions used in this paper to obtain an optimal estimator.

\section{Acknowledgement}

We thank the anonymous reviewers for providing us with their so appreciated comments, corrections and recommendations.

\section{References}

1. Achcar J.A and Leonardo R.A (1998) : Use of Markov chain Monte-Carlo methods in a Bayesian analysis of the Block and Basu bivariate exponential distribution, Annals of the Institute of Statistical Mathematics, 50,403-416.

2. Ahmed, S.E., Castro-Kuriss, C., Leiva, V., Sanhueza, A. (2010). A truncated version of the Birnbaum-Saunders distribution with an application in financial risk, Pakistan Journal of Statistics, 26(1), 293-311

3. Aouf, F. and Chadli, A. (2017). Bayesian Estimations in the Generalized Lindley Model, International journal of mathematical models and methods in applied sciences, 11, 26-32.

4. Balakrishnan, N. and Mitra, D. (2012). Left truncated and right censored Weibull data and likelihood inference with an illustration, computation statistics and data analysis.56 (12), 4011-4025.

5. Boudjerda, K., Chadli, A., Fellag, H. (2016). Posterior Analysis of the Compound Truncated Weibull Under Different Loss Functions for Censored Data, international journal of mathematics and computers in simulation, Vol 10, 265-272.

6. Chadli, A.; Talhi, H., Fellag, H. (201 3). Comparison of the Maximum Likelihood and Bayes Estimators of the parameters and mean Time Between Failure for bivariate exponential Distribution under different loss functions, Afr. Stat., Vol. 8, pp. 499-514

7. Cullen, M. J., Walsh, J., Nicholson, L. V. B., and Harris, J. B. (1990). Ultrastructural localization of dystrophin in human muscle by using gold immunolabelling. Proceedings of the Royal Society of London, Series B 20, 197-210.

8. Ghitany, M.E., Atieh, B., Nadarajah, S. (2008). Lindley distribution and its application. Mathematics and computers in simulation, 78(4), 493-506

9. Jeffreys H, (1961), Theory of probability, Oxford: Clarendon press.

10. Lawless, J. F. (2011). Statistical models and methods for lifetime data. John Wiley and Sons.

11. Lindley, D.V. (1958). Fiducial distributions and Bayes Theorem. Journal of the Royal Statistical Society. Series B (Methodological), 102-107.

12. Maha A. Aldahlan, and al.(2020) The Truncated Cauchy Power Family of Distributions with Inference and Applications. Entropy 2020, 22(3), 346. 
13. Mathews JNS, Appleton DR (1993) An application of the truncated Poisson distribution to Immunogold assay. Biometrics 49(2), 617-621.

14. Mazucheli, J., Achcar, J. A. (2011). The Lindley distribution applied to competing risks lifetime data. Computer Methods and Programs in Biomedicine 104 (2), 189-192.

15. Messaadia, H. And Zeghdoudi, H. (2018), Zeghdoudi distribution and its applications, Int. J. Computing Science and Mathematics, Vol. 9, No. 1, pp. 58-65.

16. Pitman, E. (1937). The closest estimates of statistical parameters. Mathematical proceeding of the Cambridge philosophical society, 33(2).

17. Rashad A. R. Bantan. And al.(2019), Truncated Inverted Kumaraswamy Generated Family Of Distributions with Applications. Entropy 2019, 21(11), 1089

18. Singh, S. K., Singh, U., Sharma, V. K. (2014). The Truncated Lindley Distribution: Inference and Application, Journal of Statistics Applications and Probability, 3(2), 219-228.

19. Varadhan, R and Gilbert, P. (2010). An R package for solving a large system of nonlinear equations and for optimizing a high-dimensional nonlinear objective function, Journal of statistical software, $32(4)$.

20. Zeghdoudi, H. And Nedjar, S. (2016a), Gamma Lindley distribution and its application, Journal of Applied Probability and Statistics, Vol. 11 No. 1, pp.129-138.

21. Zeghdoudi, H. And Nedjar, S. (2016b), On gamma Lindley distribution: properties and simulations, Journal of Computational and Applied Mathematics, Vol. 298, pp. 167-174.

22. Zeghdoudi, H. And Nedjar, S. (2016c), a pseudo Lindley distribution and its application, Afr. Stat., Vol. 11 No, 1, pp. 923-932. 